\title{
Rehabilitation Engineering Laboratory Research Program: Past, Present and Future
}

\author{
Milos R. Popovic \\ Institute of Biomaterials and Biomedical Engineering, University of Toronto \\ 164 College Road, Toronto, Ontario, M5S 3E, Canada \\ milos.popovic@utoronto.ca
}

\section{Extended Abstract}

In this lecture the basic concepts of the functional electrical stimulation therapy (FEST) and how it was developed will be presented. Following that we will discuss different FEST modalities and how this therapy could be used to improve reaching, grasping and walking functions in patients with various neurological deficits. At the end of this presentation segment we will discuss other potential applications of the FEST that are presently being developed and tested in our laboratory.

The second part of the presentation will be focused on brain machine interfaces (BMIs). Specifically, we will present a few BMI systems that our team developed already, and how they can be deployed clinically. We will also discuss the challenges facing the development of such technologies and why it is extremely difficult to develop clinically viable BMIs. Following that we will discuss how BMI system can be made user friendly and where we think one should expect first BMI systems to find clinical applications.

The last part of the presentation will be used to discuss selected projects that our team is presently pursuing and are applying electrical stimulation technology as means to generate devices that are able to cure diseases. Specifically, we will discuss how electrical fields can be used to navigate stem cells and how they could be used to help cure wounds that do not heal. 